\title{
Biological Immune System Applications on Mobile Robot for Disabled People
}

\author{
Songmin Jia, Xue Zhao, Wenbin Qu, and Yuchen Li \\ College of Electronic Information \& Control Engineering, Beijing University of Technology, Beijing 100124, China \\ Correspondence should be addressed to Xue Zhao; xiaoxiaoerxue@163.com
}

Received 9 July 2014; Revised 8 October 2014; Accepted 15 October 2014; Published 11 November 2014

Academic Editor: Shahram Payandeh

Copyright (C) 2014 Songmin Jia et al. This is an open access article distributed under the Creative Commons Attribution License, which permits unrestricted use, distribution, and reproduction in any medium, provided the original work is properly cited.

\begin{abstract}
To improve the service quality of service robots for the disabled, immune system is applied on robot for its advantages such as diversity, dynamic, parallel management, self-organization, and self-adaptation. According to the immune system theory, local environment condition sensed by robot is considered an antigen while robot is regarded as B-cell and possible node as antibody, respectively. Antibody-antigen affinity is employed to choose the optimal possible node to ensure the service robot can pass through the optimal path. The paper details the immune system applications on service robot and gives experimental results.
\end{abstract}

\section{Introduction}

For recent years, the robots have already massively been applied in many fields. Service robots especially have developed rapidly to complete tasks for the humanity's beneficial services. Recently, with the aging problem of the global community being increasingly serious, the service robots mainly for the elderly and the disabled have been a hot research focus. Li et al. [1] developed seven degrees of freedom movable nursing robot taking high paraplegia as the nursing object to help the patients fetch medicine, water, and books in nobody situations. Zhihua et al. [2] had presented a movable service robot with double working arms to serve the elderly and the disabled. For these service robots, path planning is an important issue. In large-scale environment with obstacles, path planning is to make the robot move along the optimal path and evade obstacles from a start position to the target location. The research of robot path planning was started at the middle of the 1960s. The interests in this area grew rapidly after the publication of Wesley [3] in 1979. From then on, many methods have been developed. There are many traditional methods, such as grid theory [4], potential field method [5], the genetic algorithm [6], and neural network [7]. Nowadays, immune system is receiving more attention and is realized as a new research hotspot of biologically inspired computational intelligence approach after the genetic algorithms, neural networks, and evolutionary computation in the research of intelligent systems [8]. It is now widely used in the fields such as data mining, network security, pattern recognition, learning, and optimization for the immune system has lots of appealing features such as diversity, dynamic, parallel management, self-organization, and self-adaptation.

In this paper, biological system is applied on mobile robot to serve the disabled with better path. To ensure the service robot can pass through the optimal path, the robot needs to move along an optimal path. Firstly, admissible space tree is generated to obtain possible nodes. Then immune system is applied for its appealing features. Referring to the immune system theory, local environment information is counted as antigen and the possible node is regarded as antibody. On this basis, the affinity is calculated to choose the optimal possible node. Finally, with the node update rules, the optimal path is planned.

The remainder of this paper is organized as follows. In Section 2, the overall design is addressed. Section 3 presents biological immune system in detail. Section 4 describes the application of immune system on mobile robot followed by experiment results given in Section 5. Conclusions and future work are discussed in Section 6. 


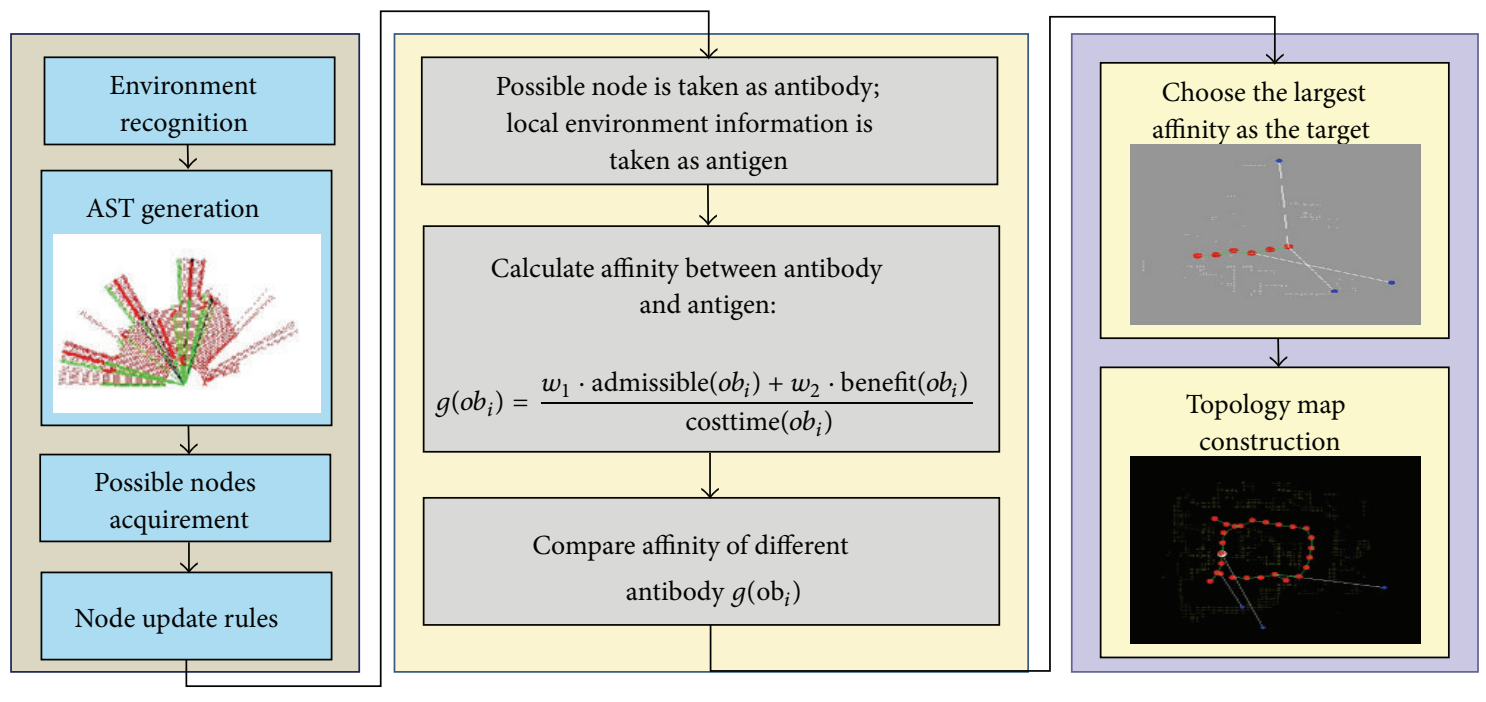

FIGURE 1: Details of immune system application on mobile robot.

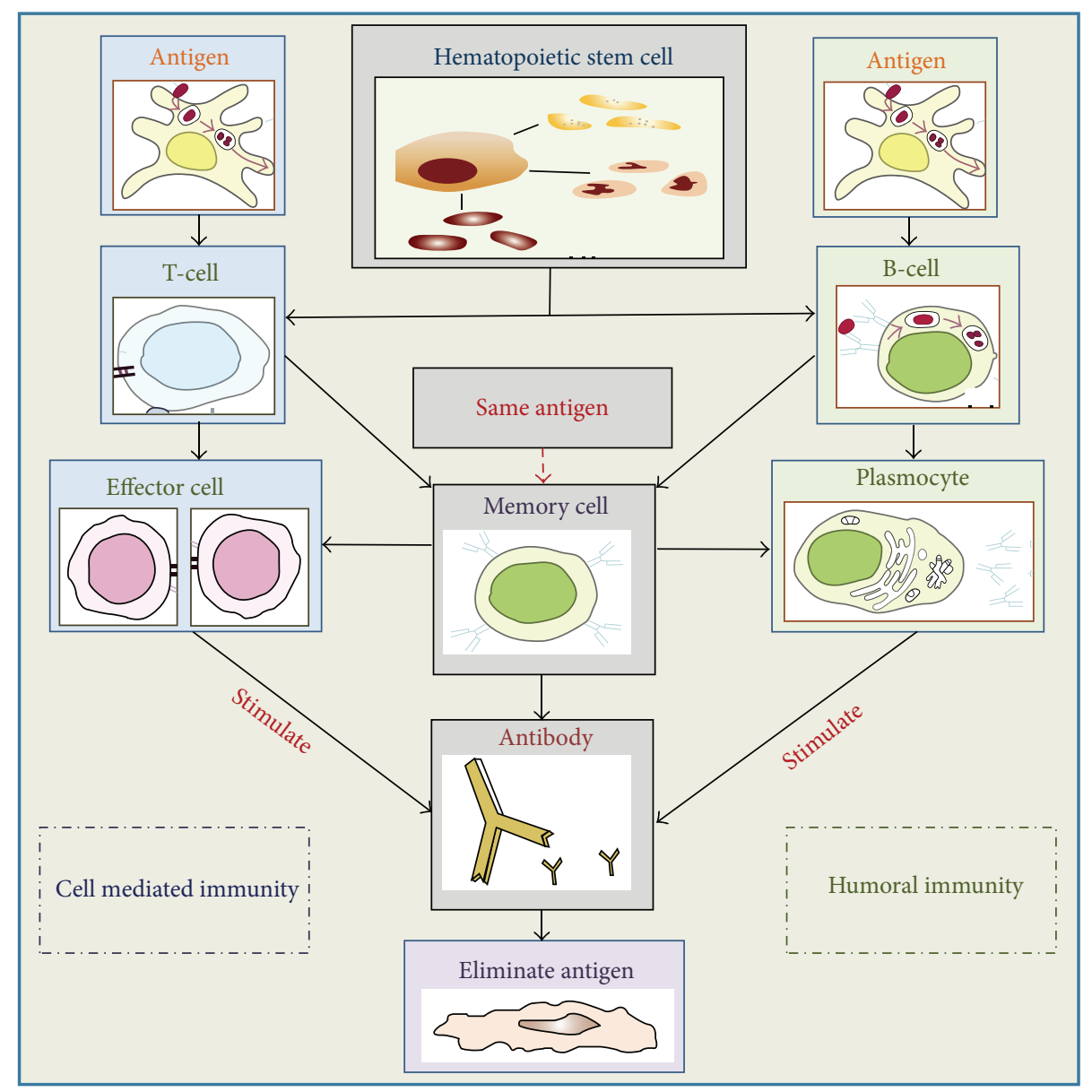

FIgURE 2: Abstract model of immune system. 


\section{Overall Design for Immune System Application on Mobile Robot}

To serve the disabled well, immune system is applied on mobile robot for its advantages such as self-organization, parallel management, self-adaptation, diversity, and dynamic. The detail of application is shown in Figure 1.

Broadly speaking, to accomplish the serving task, this proposed method can be divided into three parts. One is admissible space tree generation. The purpose of this part is to find possible nodes for robot. Another one is immune system application. This part aims to optimize possible nodes; thus the robot path can be optimal during serving process. Last one is topology map construction. For this part, topology map is constructed to demonstrate the path which the robot has passed.

\section{The Biological Immune System Theory}

The biological immune system, which protects living bodies from the invading of foreign substances such as viruses, bacteria, and other parasites (called antigens), has two types of immunity in the human body. One is the humoral immunity and the other one is the cell mediated immunity.

Two main types of lymphocyte, namely, B-cell and T-cell, play a remarkable role in both immunities [9]. B-cell participates in the humoral immunity with secreting antibodies by the clonal proliferation and $\mathrm{T}$-cell takes part in cell mediated immunity. For T-cells, there are two classes. One class is called killer T-cells, which destroy the infected cell when the infection is recognized. The other class is called helper T-cells, which trigger clonal expansion and stimulate or suppress antibody formation. Lymphocytes float freely in the blood and lymph nodes to patrol for foreign antigens. When an infectious foreign pathogen attacks the human body, the lymphocytes will be sensitive to these antigens and become activated. Then the helper T-cell releases the cytokines, which are the proliferate signals acting on the producing B-cell or the other remote cells. On the other hand, B-cell becomes stimulated and creates antibodies when a B-cell recognizes an antigen. The secreted antibodies are the soluble receptors of B-cell and these antibodies can be distributed throughout the body. An antibody's paratope can bind with an antigen's epitope according to their affinity. Moreover, an antibody can not only distinguish an antigen, but also be distinguished by other antibodies [10]. The biological immune system abstract model is illustrated in Figure 2.

\section{Immune System Application on Robot}

With the aging problem of the global community being increasingly serious, the service robots mainly for the elderly and the disabled have been paid more attention to. Therefore, path planning, as a key issue for serving task, has been a hot research topic. Meanwhile, biological immune system is receiving more attention and is widely applied in many fields for advantages of diversity, dynamic, parallel management, self-organization, and self-adaptation. To meet the elderly and the disabled demands and serve delivery tasks well, immune system is applied on robot with its specific advantages. According to the biological immune system, possible nodes are regarded as antibodies and local environment information is considered an antigen. Affinity between antibody and antigen is the chosen reference of target node to ensure the robot path is optimal throughout the delivery task service.

4.1. Possible Nodes. Admissible space tree, a set of nodes and graphs, is presented to demonstrate that the robot can move from current position to the nodes directly in the environment. The gathered reading data from laser scanning is separated into several layers according to the Euclidean distance far from the robot. Some of layers are usually partitioned into different sections since the existence of obstacles. At current stage, there are two approaches that can be used to obtain the admissible space tree of the robot. One requires the area covered with obstacle to be dilated, and whether a section is wide enough to pass the robot will be accordingly discriminated. The other one uses the width of the robot as measurement criterion in selection of admissible space tree [11].

When the current AST is available, the furthest node is selected as current possible node $P_{j}^{c}$, which describes the admissible direction. A set of possible nodes $P_{c}$ is established for the current node. It is defined as follows:

$$
P_{c}=\left\{P_{j}^{c}\right\} \text {. }
$$

While the robot is exploring in the unknown environment autonomously, possible nodes are generated continuously. Sometimes, some of those nodes closed to the established topology or possible nodes may not belong to a former topology's admissible space tree.

Therefore, in order to avoid revisiting and guarantee the global convergence of instant goal node, node updating rules are used to append, rebind, or get rid of those nodes. The details of the possible nodes chosen process and node updating rules are illustrated in Figure 3.

4.2. Immune System Application. In the biological immune system, the antibody can recognize antigen based on affinity. Immune response will produce when the affinity between antibody and antigen is large. Through combining with each other, binding is formed and the antigen is promoted to be apoptotic gradually.

To apply immune system on service robot, the service robot is counted as B-cell, possible node is considered an antibody, and local environment information is regarded as an antigen. For biological immune system, the larger the affinity is, the more benefits the body has. So following the immune system theory, affinity of service robot system, which is defined as $g\left(o b_{i}\right)$, is calculated as follows:

$$
g\left(o b_{i}\right)=\frac{w_{1} \cdot \text { obstacle }\left(o b_{i}\right)+w_{2} \cdot \text { benefit }\left(o b_{i}\right)}{\text { costtime }\left(o b_{i}\right)},
$$

where $w_{1}, w_{2}$ mean harmonic parameters. benefit $\left(o b_{i}\right)$ is defined as forecast ability of environmental information 


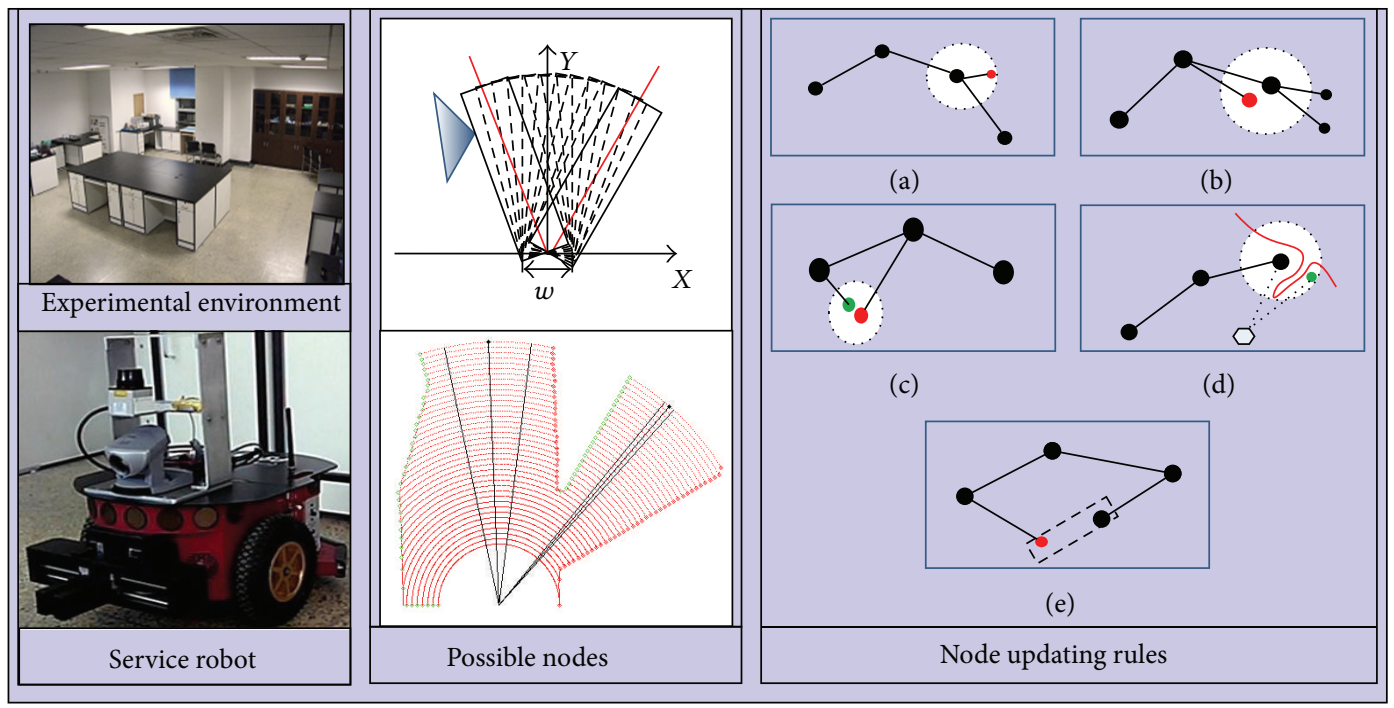

Figure 3: Possible nodes abstraction process.

acquirement. It represents unknown grid unit number in the circle area with the antibody as center and laser scan range as radius. During the delivery task, the more environmental information the exploration environment includes, the larger the exploration worth is. obstacle $\left(o b_{i}\right)$ is defined as exploration attractor when the robot moves to the obstacle; it is usually valued from one to ten. The higher the value is, the more the occupied grid number is. This is one fixed thought based on autonomous exploration, which means the area nearby the obstacle has much more environmental information. Meanwhile, costtime $\left(o b_{i}\right)$ stands for the time cost in the robot moving from current position to the corresponding possible node. In conclusion, the larger the parameter $g\left(o b_{i}\right)$ is, the better the robot can serve. So, the possible node with a larger affinity will be chosen as target node where the robot will move.

\section{Experimental Results}

To verify the good performance of path planning brought by immune system application, some experiments are conducted.

5.1. Experiments on Robot. The experiment on robot is carried out in the experimental environment. The experiment results are demonstrated in Figure 4.

Figure 4(a) illustrates the experimental environment and Figure 4(b) shows robot positions at four moments. Moreover, Figure 4(c) describes the possible nodes abstraction process and Figure 4(d) illustrates the topological map. The red nodes donate topology nodes, which mean the positions the robot passed during delivery service. Blue nodes are possible nodes.

To illustrate advantages brought by introducing immune system, comparative experiment without immune system introduced is described in Figure 5(a), and the exploration result with immune system is illustrated in Figure 5(b).
As shown in Figure 5(a), the robot explored areas repeatedly in the rectangles surrounded by blue lines, while the exploration performance of robot with immune system in Figure 5(b) is obviously better.

5.2. Experiments on Wheelchair. The path planning strategy based on immune system is applied on the wheelchair platform to verify the practicality. The experiment results are demonstrated in Figure 6.

Figure 6(a) shows the experiment and the path of wheelchair and Figure 6(b) shows the four moments of wheelchair movements.

From the results, we can see that the wheelchair can avoid obstacles and move in an optimal path using our path planning strategy based on immune system.

In conclusion, with the path planning strategy based on immune system, service robot can do delivery task and make the disabled and the elderly movement more convenient and so on.

\section{Conclusion}

To serve the elderly and the disabled better, the service robot must move through an optimal path. So immune system is applied on service robot for its advantages.

Firstly, the robot abstracts admissible space tree to find possible nodes. Then, compared with immune system, possible node is regarded as antibody and local environmental information is considered an antigen. Finally, experiments on robot and wheelchair are conducted to verify practicality of our path planning strategy.

Further endeavor will be made to investigate the improvement of current algorithm and will focus on multirobotic system in the future. 


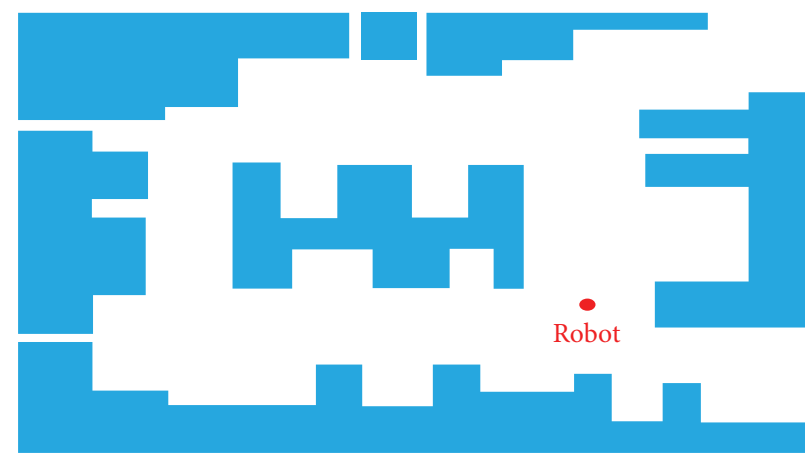

(a) Experimental environment

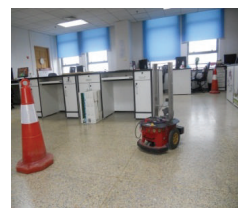

(1)

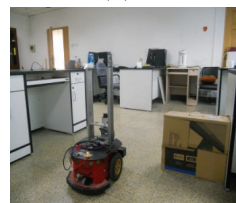

(3)

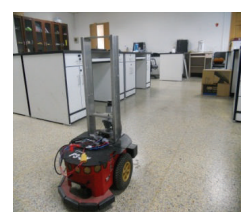

(2)

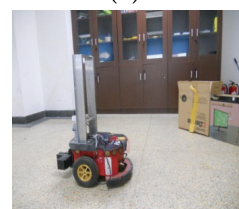

(4)

(b) Robot positions at four moments

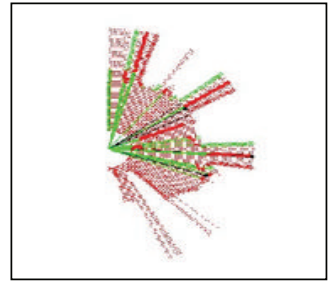

(1)

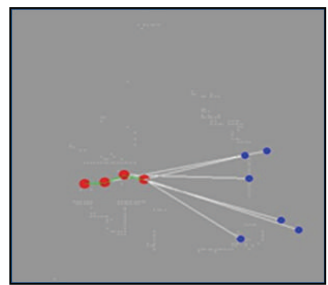

(1)

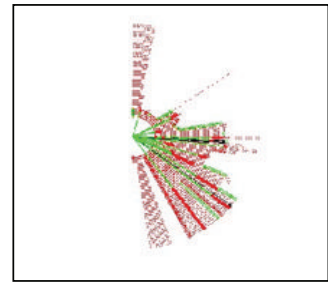

(2)

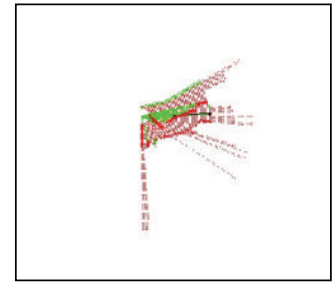

(3)

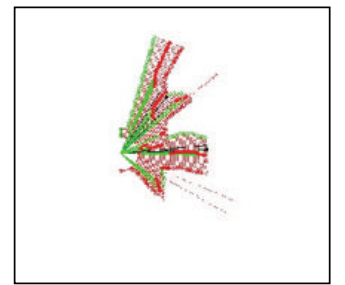

(4)

(c) Possible nodes abstraction

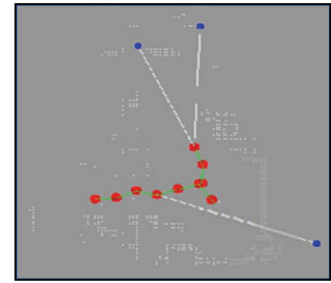

(2)

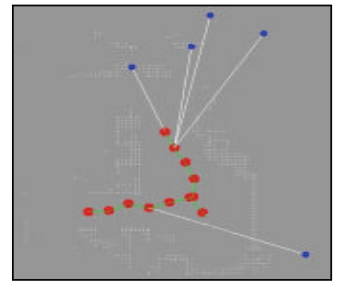

(3)

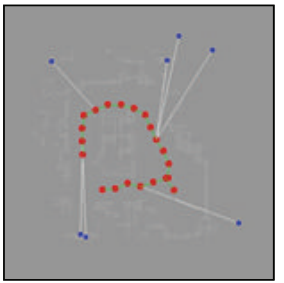

(4)

(d) Topology map construction

FIGURE 4: Experiment on robot.

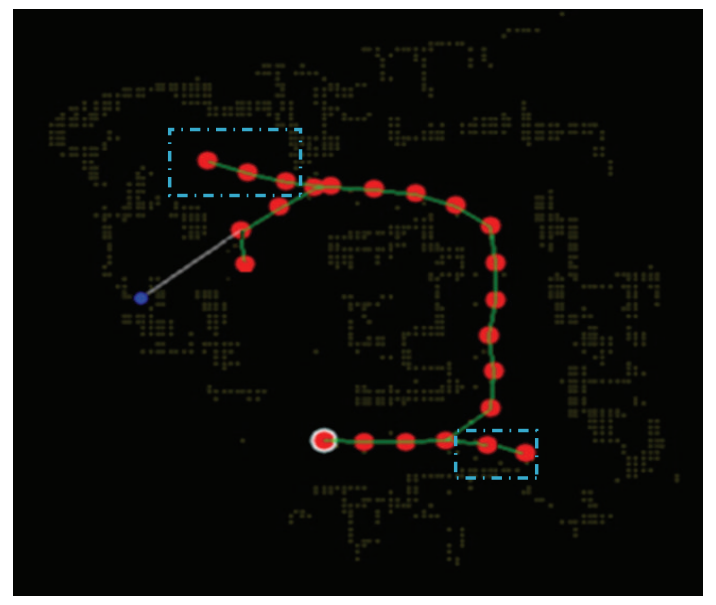

(a) Environment exploration without immune system

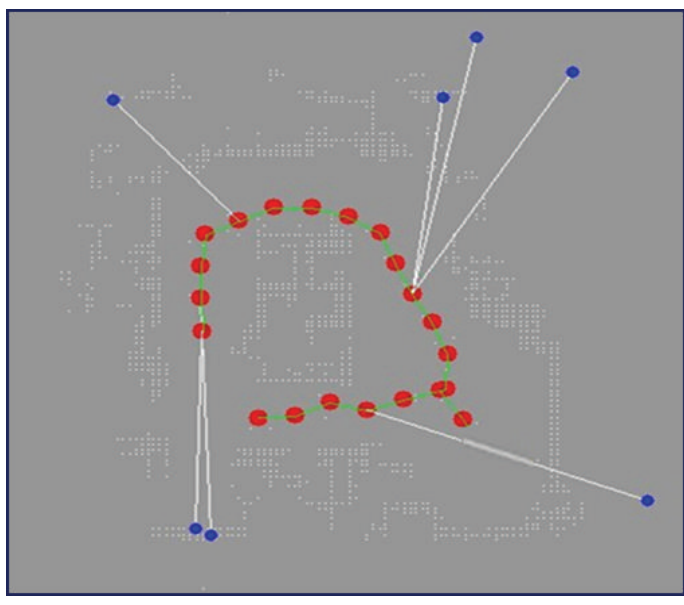

(b) Environment exploration with immune system

FIGURE 5: Comparative experiments. 


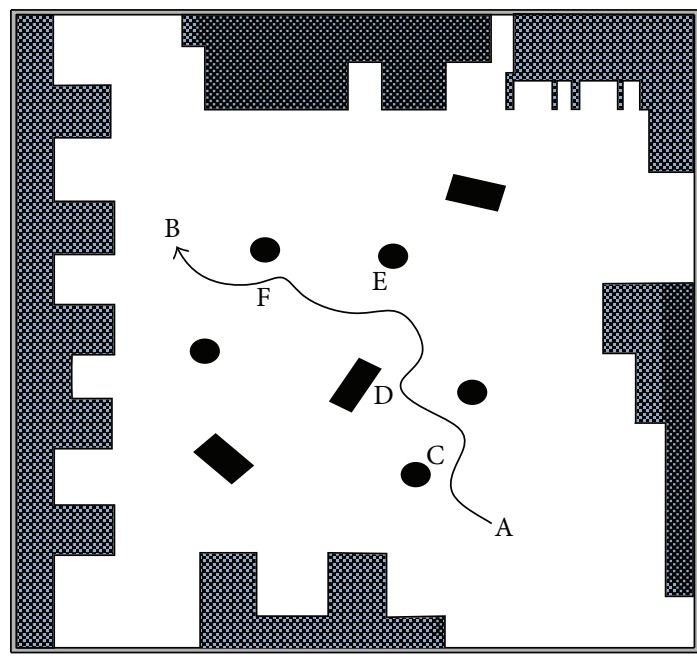

(a) Environment and path

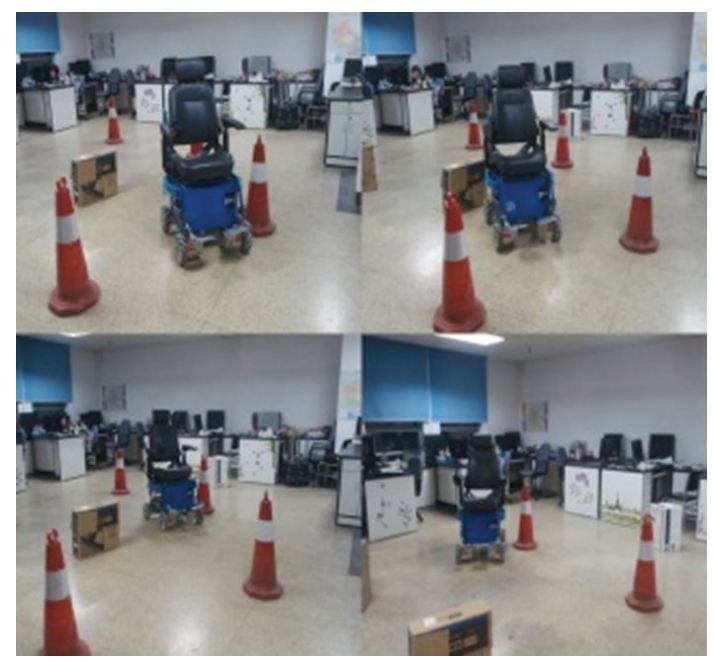

(b) Wheelchair movement

FIGURE 6: Experiment on wheelchair.

\section{Conflict of Interests}

The authors declare that there is no conflict of interests regarding the publication of this paper.

\section{Acknowledgments}

This research work is supported by Key Program of Beijing Natural Science Foundation (KZ201110005004) and National Natural Science Foundation (61175087 and 61105033).

\section{References}

[1] J. Li, J. Zhang, and D. Jin, "Development of a mobile health care robot," Chinese Journal of Rehabilitation Medicine, vol. 10, no. 3, pp. 126-128, 1995 (Chinese).

[2] Z. Zhihua, Z. Jianyong, L. Yang, and L. Gang, "Development of a movable service robot with double working arms for the elderly and the disabled," in Proceedings of the International Conference on Electronic Computer Technology (ICECT '09), pp. 636-640, February 2009.

[3] M. A. Wesley, "An algorithm for planning collision-free paths amoung polyedral obstacles," Communications of ACM, vol. 2, pp. 959-962, 1979.

[4] Y.-S. Liu, N. Wei, and Y.-M. Sun, "Path planning algorithm based on grid method for virtual human," Computer Engineering and Design, vol. 29, no. 5, pp. 1229-1230, 2008.

[5] D. Gu, H. Hu, J. Reynolds, and E. Tsang, "GA-based learning in behaviour based robotics," IEEE Transactions on Computational Intelligence in Robotics and Automation, vol. 3, pp. 1521-1526, 2003.

[6] G. Chen and L. Shen, "Genetic path planning algorithm under complex environment," Robot, vol. 23, no. 1, pp. 40-43, 2001.

[7] J. Yu, V. Kromov, Z. Sun, and H. Narihisa, "Fast algorithm for path planning based on neural network," Robot, vol. 23, no. 3, pp. 201-205, 2001.

[8] X. Hu, C. Xie, and Q. Xu, "Robot path planning based on artificial immune network," in Proceedings of the IEEE International
Conference on Robotics and Biomimetics (ROBIO '07), pp. 10531057, Sanya, China, December 2007.

[9] R. O. Canham and A. M. Tyrrell, "A hardware artificial immune system and embryonic array for fault tolerant systems," Genetic Programming and Evolvable Machines, vol. 4, no. 4, pp. 359-382, 2003.

[10] D. Dasgupta, "Artificial neural networks and artificial immune systems: similarities and differences," in Proceedings of the IEEE International Conference on Systems, Man, and Cybernetics, pp. 873-878, Orlando, Fla, USA, 1997.

[11] S. S. Ge, Q. Zhang, A. T. Abraham, and B. Rebsamen, "Simultaneous path planning and topological mapping (SP2ATM) for environment exploration and goal oriented navigation," Robotics and Autonomous Systems, vol. 59, no. 3-4, pp. 228-242, 2011. 

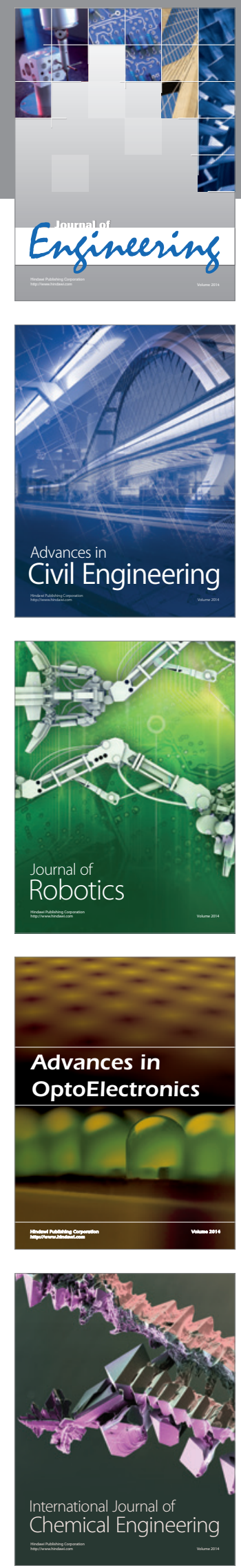

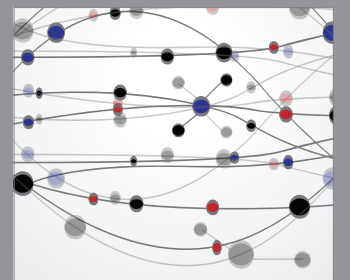

The Scientific World Journal
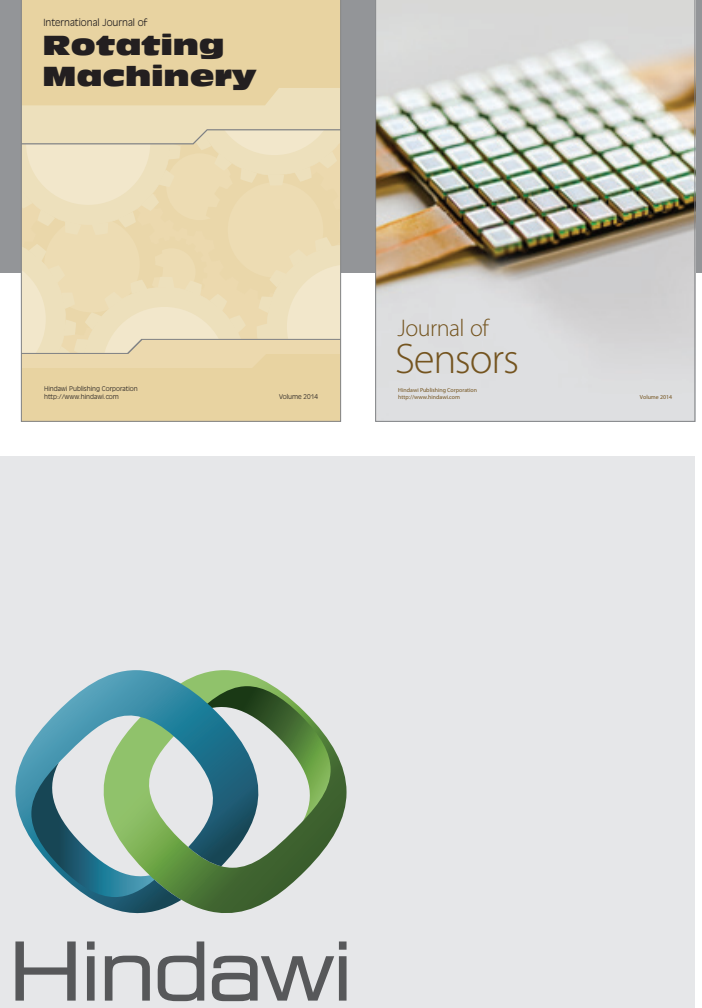

Submit your manuscripts at http://www.hindawi.com
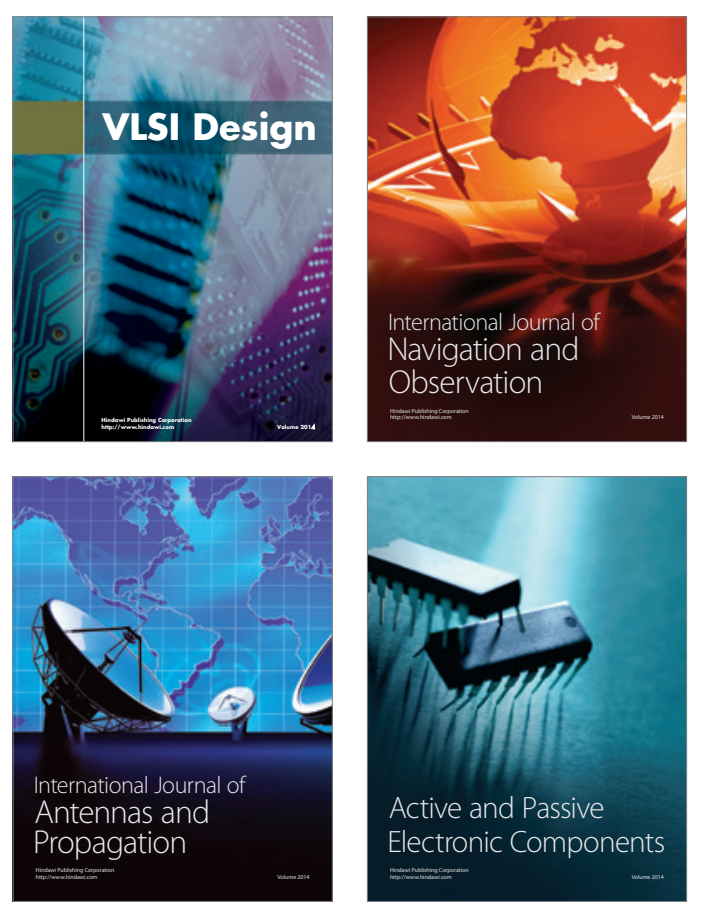
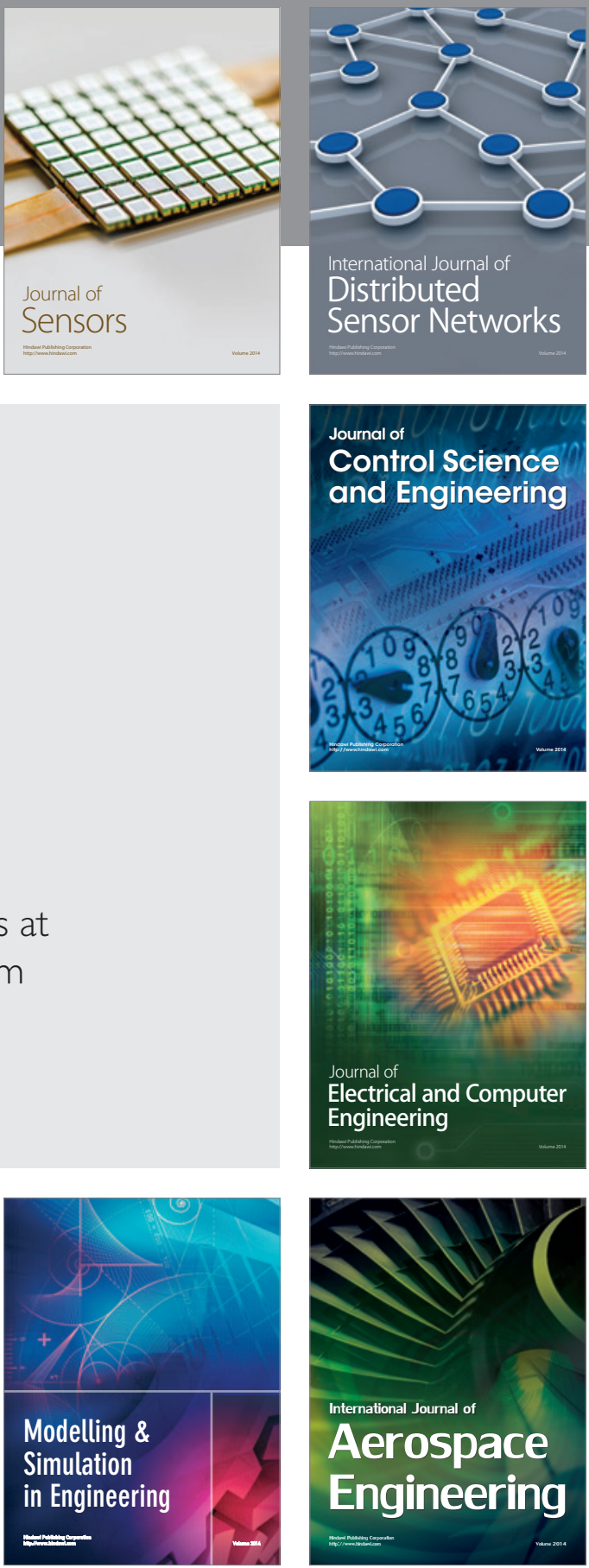

Journal of

Control Science

and Engineering
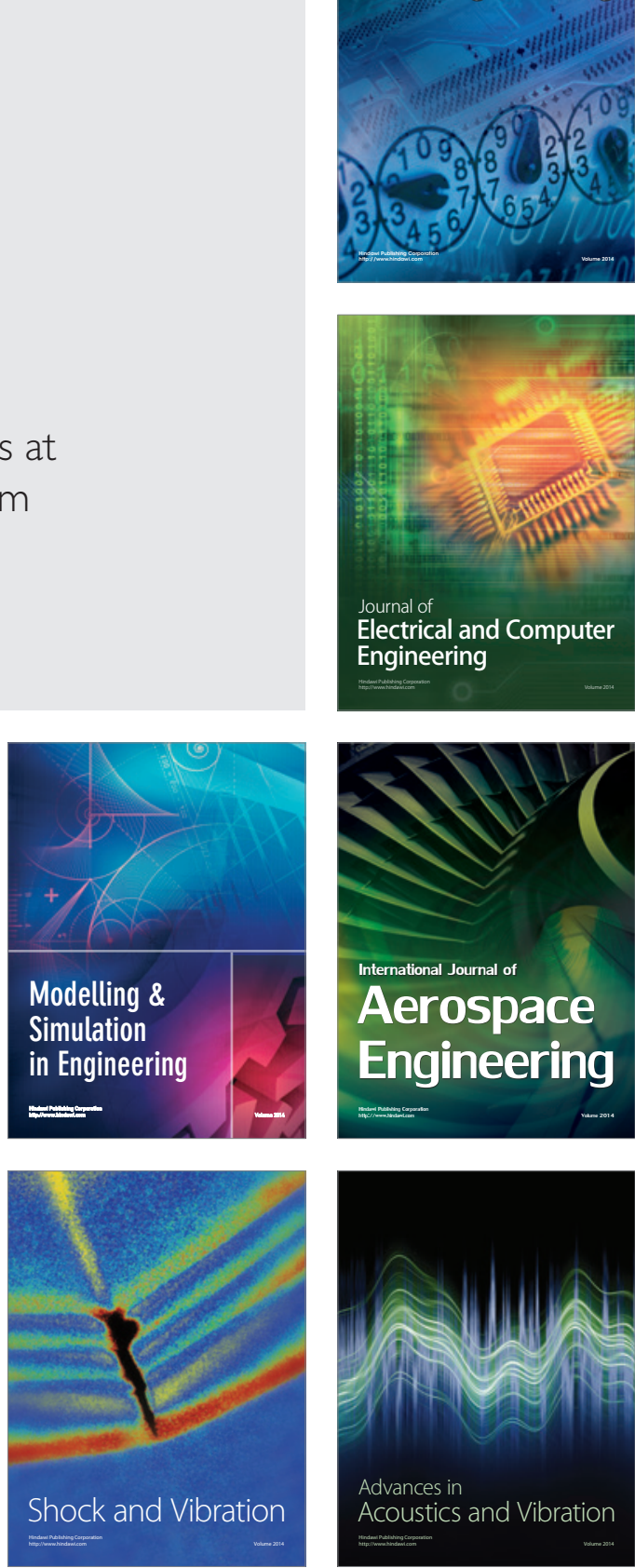